\title{
Nuclear structure effects on heavy-ion reactions with microscopic theory
}

\author{
K. Vo-Phuoc ${ }^{1, a}$, C. Simenel ${ }^{1}$, and E. C. Simpson ${ }^{1}$ \\ ${ }^{1}$ Department of Nuclear Physics, Research School of Physics and Engineering, Australian National University, Canberra, ACT \\ 2601, Australia
}

\begin{abstract}
The self-consistent mean-field Hartree-Fock (HF) theory, both static and time-dependent (TDHF) versions, is used to study static and dynamic properties of fusion reactions between even ${ }^{40-54} \mathrm{Ca}$ isotopes and ${ }^{116} \mathrm{Sn}$. The bare nucleus-nucleus potential, calculated with the frozen HF approach, is affected by the groundstate density of the nuclei. However, once dynamical effects are included, as in TDHF, the static effects on the barrier are essentially washed out. Dynamic properties of the nuclei, including low-lying vibrational modes, are calculated with TDHF and selectively used in coupled-channels calculations to identify which modes have the most effect on the TDHF fusion threshold. Vibrations cannot fully explain the difference between the static HF and TDHF fusion barriers trend so other dynamical effects such as transfer are considered.
\end{abstract}

\section{Introduction}

Nuclear structure plays a major role in nuclear fusion reactions. Effects of internal nuclear structure on heavy-ion fusion can be seen by studying features of experimental fusion barrier distributions [1, 2]. Experiments have shown that dynamic effects such as low-lying vibrational couplings [3-5] and transfer reactions [6-10] strongly affect fusion reactions. Other dynamic effects, such as breakup [11-14] and rotational couplings [4], have also been seen to play a role.

Different theoretical approaches can be used to study fusion. Conventionally, the coupled-channels approach is applied to study reactions and compare fusion cross sections with experimental data, for example, but it requires input parameters of the structure of the nuclei in the reaction. When these parameters are not known or where experimental data is not available, a more microscopic theoretical method is valuable.

The Hartree-Fock (HF) method is a self-consistent theory which is a useful tool to study both nuclear structure and reactions as its only input is the nucleon-nucleon interaction. The time-dependent version (TDHF) allows for the study of dynamical effects on fusion reactions. TDHF has been used for several purposes for nuclear structure and reactions (see Refs. [15, 16] for reviews). Modern codes solve the TDHF equation in three dimensions and include spin-orbit interaction [17-20]. Recent applications include vibration [21-25], fusion [26-30], transfer [31-35], quasifission [36-38] and fission [39-42] studies.

TDHF includes many dynamical effects simultaneously and it is difficult to isolate the effect of a single dynamical coupling on a fusion reaction. Here the method of including microscopic inputs to coupled-channels (CC)

\footnotetext{
a e-mail: kirsten.vo-phuoc @anu.edu.au
}

analysis [27] is used to help unravel the TDHF dynamics and the influence of these on the fusion reactions.

\section{Static effects}

\subsection{Numerical details}

There are two steps to obtaining a nucleus-nucleus potential using the frozen HF method. The first step is the calculation of an individual HF ground state nucleus wavefunction. This was done for all the projectiles ${ }^{40-54} \mathrm{Ca}$ and target ${ }^{116} \mathrm{Sn}$. The HF method assumes that nucleons in a nucleus are independent particles and that they generate a self consistent mean-field. The ground state nucleus wavefunction for each nucleus was calculated using the ev8 code [43]. The HF equation

$$
[h[\rho], \rho]=0,
$$

where $\rho$ is the one-body density matrix of the independent particle system and $h[\rho]$ is the HF hamiltonian, is solved on a $3 \mathrm{D}$ grid on a box size $(2 \times 11.2)^{3} \mathrm{fm}^{3}$ with three planes of symmetry. The effective nucleon-nucleon interaction was taken to be the Skyrme interaction [44] with the SLy4d [17] parametrisation. This parametrisation ignores centre of mass corrections and because of this it can be used for TDHF calculations of heavy-ion collisions as well $[16,45]$. Pairing is accounted for at the BCS level [46].

The second step is then the calculation of the HF bare nucleus-nucleus potential between two nuclei, also known as the frozen HF potential. This is done by taking two HF ground states for the reaction and putting them in the same calculation box. In this work all frozen HF calculations were made in a box size $67.2 \times 22.4 \times(2 \times 11.2) \mathrm{fm}^{3}$ in the $x-y-z$ orientation ( $z=0$ is a plane of symmetry) where 


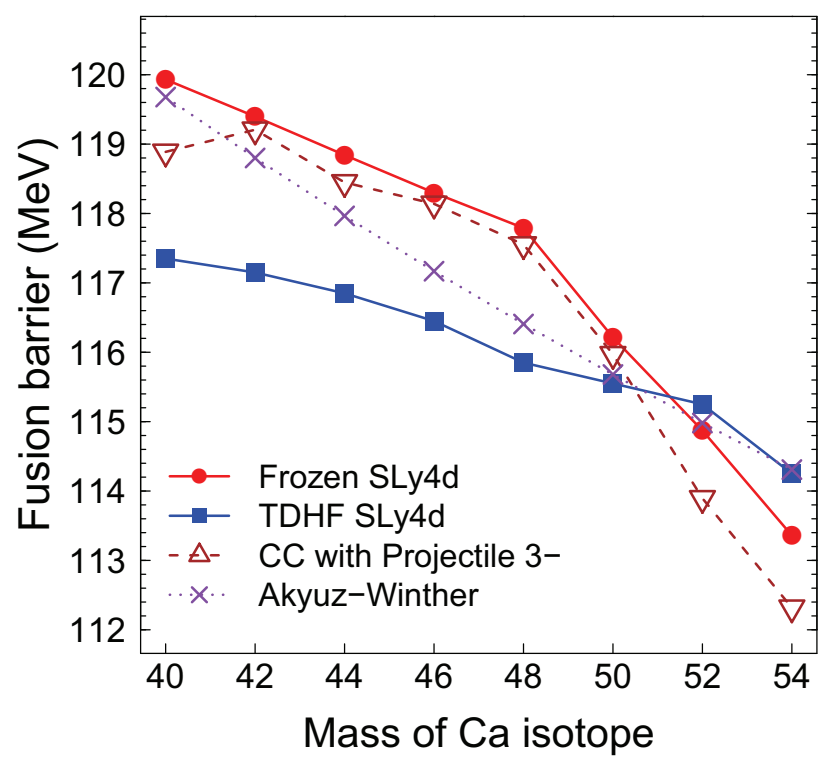

Figure 1. Fusion barriers for ${ }^{A} \mathrm{Ca}+{ }^{116} \mathrm{Sn}$ obtained with the frozen HF (circles), TDHF (squares), coupled-channel (triangle) and Akyuz-Winther (crosses) approaches.

the collision axis is the $x$-axis. The maximum distance between the centres of masses was $44.8 \mathrm{fm}$. For every distance between the centres of masses, $\mathbf{r}$, the frozen $\mathrm{HF}$ potential $V(\mathbf{r})$ is calculated. Using the HF ground state density distributions $\rho_{1}$ and $\rho_{2}$ of the nuclei, the nuclear part of the potential, $V_{N}(\mathbf{r})$, is the sum of the energies

$$
V_{N}(\mathbf{r})=E(\mathbf{r})-E_{\mathrm{HF}}\left[\rho_{1}\right]-E_{\mathrm{HF}}\left[\rho_{2}\right],
$$

for the energy of the total system

$$
E(\mathbf{r})=\int \mathcal{E}\left[\rho_{1}\left(\mathbf{r}^{\prime}\right)+\rho_{2}\left(\mathbf{r}-\mathbf{r}^{\prime}\right)\right] d \mathbf{r}^{\prime},
$$

and the (HF) energy for each nucleus

$$
E_{\mathrm{HF}}\left[\rho_{j}\right]=\int \mathcal{E}\left[\rho_{j}(\mathbf{r})\right] d \mathbf{r},
$$

where $\mathcal{E}$ is the Skyrme energy density that arises from the Skyrme interaction.

\subsection{Static fusion barriers}

Fusion barrier energies as obtained by frozen $\mathrm{HF}$ are shown in Fig. 1 (circles) alongside the fusion barriers obtained from the Akyüz-Winther potential [47] (crosses). The latter shows a near constant decrease of the fusion barrier energy, $V_{B}$, as neutron number is increased. By contrast, the results obtained from static HF calculations show a change of gradient in the $V_{B}$ line before and after the ${ }^{48} \mathrm{Ca}$ projectile. To explain what causes this trend we can consider some static nuclear properties arising from the HF ground state calculations of the calcium projectiles. One of these properties is the root-mean-square (rms) radius. Both neutron and proton rms radii are shown in Fig. 2 . Since the tin target is constant in all reactions, the focus will be on the calcium chain. The neutron rms radius

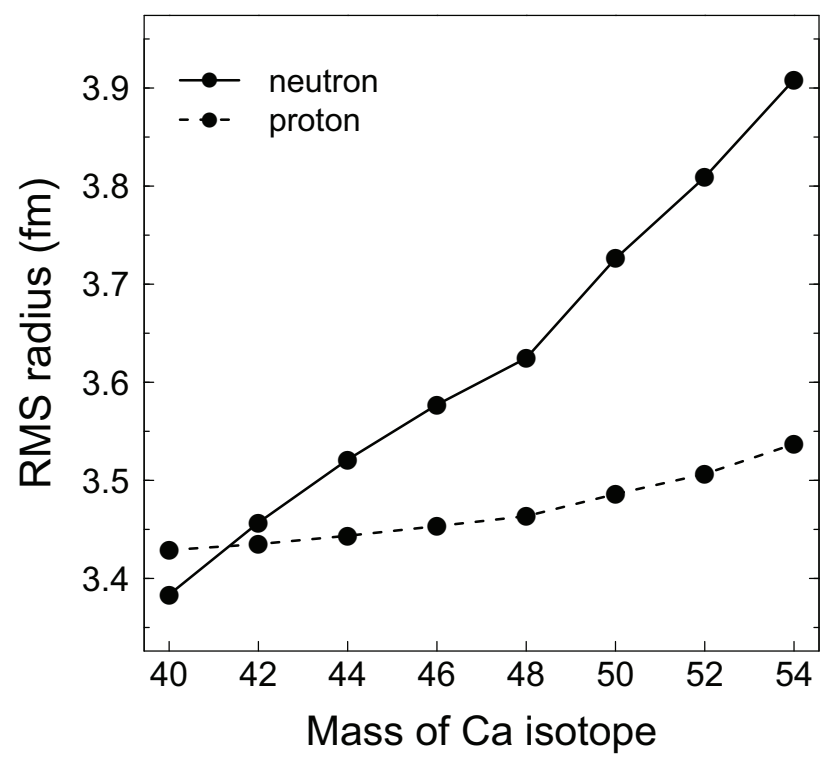

Figure 2. Calcium isotope proton (dashed line) and neutron (solid line) rms radii in the SLy4d parametrisation.

(solid line) exhibits a change of gradient before and after the ${ }^{48} \mathrm{Ca}$ nucleus. An increase in nuclear radius induces a decrease of barrier energies. This is because the point at which the fusion barrier occurs between the nuclei will be at a larger distance thereby decreasing the Coulomb potential and hence the overall fusion barrier. As to why the rms radii behave like this we must turn to single particle shells of the neutrons in the calcium nuclei. The change of gradient happens between the filling of the $1 f_{\frac{7}{2}}$ and the $2 p_{\frac{3}{2}}$ shells. As ${ }^{48} \mathrm{Ca}$ is a doubly magic nucleus the energy gap between these shells is substantial. The neutrons in the $2 p$ shells are less bound, meaning their radial wavefuncion extends to a larger radius than the neutrons in the $1 f_{\frac{7}{2}}$ shell which gives rise to a more rapid increase of neutron rms radius.

\section{Dynamic effects}

\subsection{Dynamic fusion barriers}

To account for a more realistic picture of fusion reactions, dynamical couplings must be included since nuclei do not necessarily stay in their ground state during fusion reactions. To study this with HF theory, the time-dependent version (TDHF) must be considered. The TDHF equation

$$
i \hbar \frac{d \rho}{d t}=[h[\rho], \rho]
$$

is solved by the TDHF3D code [17] with the SLy4d parametrisation of the Skyrme functional as for the static case. A mean field is now defined for the entire system, generated by all the nucleons. The single-particle occupation numbers induced by pairing correlations are kept constant in the dynamics.

To compute a fusion barrier energy for a particular system, as for the static case we again start with both the HF 
ground states in the same calculation box at some separation distance. A Galilean boost, $e^{i \mathbf{k}_{j} \cdot \hat{\mathbf{r}}}$, giving each nucleon in nucleus $j$ momentum is applied to the system at initial time which moves the nuclei towards each other. In TDHF, the fusion probability is either 0 or 1 . Here, fusion is deemed successful if a compound nucleus has formed in $5 \mathrm{zs}$. The TDHF fusion barrier, or "threshold", is found by incrementally increasing the energy for a system until fusion is observed [48].

The TDHF fusion thresholds are shown with squares in Fig. 1. We see that, including dynamics, in most cases, reduces the static fusion barrier. We also observe that including dynamics washes out the static structure effects. Indeed, there is no longer a change of gradient before and after the ${ }^{48} \mathrm{Ca}$ projectile in the TDHF results as seen for the static frozen HF barriers. The expected dynamical effects present in these TDHF calculations are vibrations and transfer. Indeed, coupling to rotational states is not expected to play a major role as the nuclei are essentially spherical. The goal is then to unravel the dynamics in the TDHF calculations to see which dynamical effects have an impact on fusion thresholds.

\subsection{Vibrations}

Low-lying octupole phonons are known to play an important role in fusion, in particular in reactions with ${ }^{40} \mathrm{Ca}$ [49]. Therefore the coupling to the $3_{1}^{-}$state is considered for all calcium isotopes. Quadrupole vibrations could also impact fusion. However, the low-lying $2^{+}$states in ${ }^{40} \mathrm{Ca}$ are not collective. The study of the couplings to $2_{1}^{+}$states in the other calcium isotopes will be the subject of future work.

Studies of vibrations of a single nucleus can be done by performing a TDHF calculation for that nucleus in isolation. An octupole boost, $e^{i \varepsilon \hat{Q}_{3}}$, with boost intensity $\varepsilon$ small enough to be in the linear regime and octupole operator

$$
\hat{Q}_{3}=\sqrt{\frac{7}{16 \pi}} \sum_{i=1}^{A}\left(2 \hat{x}^{3}-3 \hat{x}\left(\hat{y}^{2}+\hat{z}^{2}\right)\right)
$$

is applied to the HF ground state of calcium isotopes at initial time. The response of the calcium nucleus to this boost is determined by the expectation value of the octupole operator, also known as the octupole moment $\langle\hat{Q}\rangle(t)$.

The TDHF 3D code is used to compute the evolution of the octupole moment after the initial octupole boost. As the calculations involve only one nucleus, it is possible to use a Skyrme interaction which has been fitted with the center of mass corrections. To study vibrations, we therefore use the SLy4 paramaterisation [50] which includes such corrections. The octupole moment of the calcium was calculated over $15 \mathrm{zs}$ and is shown in the top panel of Fig. 3 for ${ }^{40} \mathrm{Ca}$ as an example. An oscillation is observed due to the excitation of the octupole phonon.

To resolve an energy spectrum for this, the Fourier transform is taken to produce an octupole strength func-
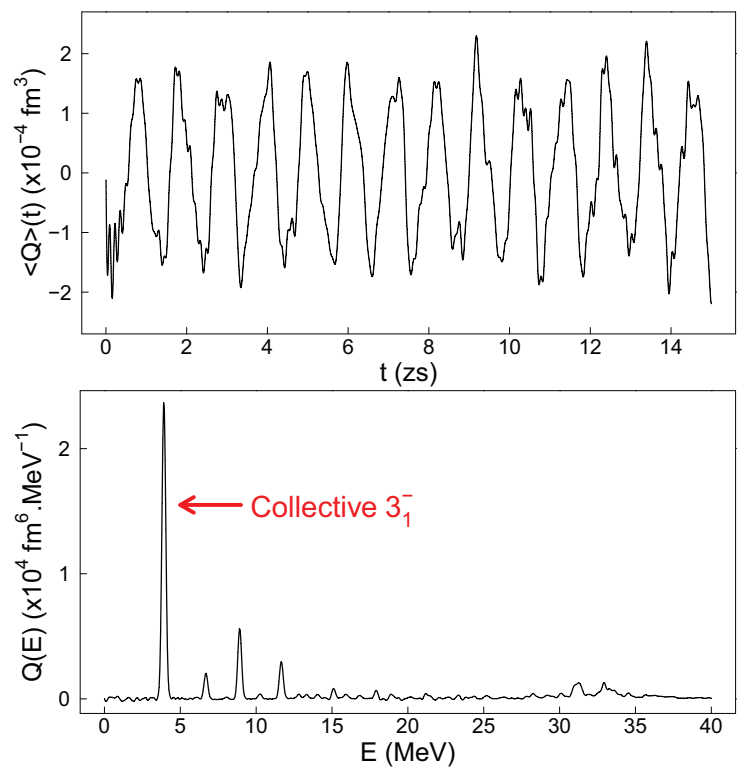

Figure 3. Evolution of the octupole moment in ${ }^{40} \mathrm{Ca}$ (top) and corresponding octupole strength function (bottom).

tion, $Q(E)$, as a function of energy (see, e.g., [24]):

$$
\begin{aligned}
Q(E) & =\frac{-1}{\pi \hbar \varepsilon} \int_{0}^{\infty} d t\langle\hat{Q}\rangle(t) \sin (E t / \hbar) \\
& \equiv \sum_{v}\left|q_{v}\right|^{2} \delta\left(E-E_{v}\right)
\end{aligned}
$$

where $q_{v}=\langle v|\hat{Q}| 0\rangle$ is the transition amplitude between the ground state $|0\rangle$ and the excited state $|v\rangle$. In practice, $\langle\hat{Q}\rangle(t)$ is multiplied by a damping function to avoid spurious oscillations in the Fourier spectrum due to the finite time of the TDHF calculation. The resulting strength function is shown in the bottom panel of Fig. 3.

The first intense peak of the strength function corresponds to the first collective $3_{1}^{-}$state. The position of the peak is at the excitation energy of the $3_{1}^{-}$state (as seen by TDHF) and the area under the peak corresponds to the transition probability $\left|q_{3}\right|^{2}$. This can be used to determine the deformation parameter $\beta_{3}$ according to [51]

$$
\beta_{3}=\frac{4 \pi\left|q_{3}\right|}{3 R_{0}^{3} A},
$$

where $R_{0}$ is the nuclear radius. The energy and deformation parameter of the $3_{1}^{-}$state in ${ }^{40} \mathrm{Ca}$ obtained with TDHF are compared with experimental values in Table 1 . Despite an underestimation of the deformation parameter, the agreement between theory and experiment is reasonable. Similar calculations of $3_{1}^{-}$properties have been computed with TDHF for the other calcium isotopes.

To see what this one dynamical effect has on the fusion barriers of the systems, the CC approach [52], using the CCFULL code [53] is used since dynamical effects can be added in one at a time. Couplings to low-lying states could induce a complex structure in the distribution of fusion barriers [2]. The main feature, however, is a lowering of the main peak in the barrier distribution, often resulting 


\begin{tabular}{lcc}
\hline & $E_{x}\left(3_{1}^{-}\right)(\mathrm{MeV})$ & $\beta_{3}$ \\
\hline Exp. & 3.737 & $0.3-0.4$ \\
TDHF & 3.916 & 0.184
\end{tabular}

Table 1. Comparison of excitation energy and deformation parameter between TDHF and experimental values [51].

in a lowering of the average barrier. These average barriers, calculated with coupling to the $3_{1}^{-}$state in the calcium isotopes, are shown in Fig. 1. As expected, this coupling lowers the barrier, in comparison to the frozen HF static barrier, in all cases. Note that couplings to low-lying $2^{+}$ states, not included here, are expected to produce a similar effect.

The inclusion of couplings to low-lying vibrations is helpful for explaining the difference between the static and dynamic cases for the systems with ${ }^{40-50} \mathrm{Ca}$ as projectiles but not for the two most neutron-rich systems. Indeed, we see in Fig. 1 that for the ${ }^{52,54} \mathrm{Ca}+{ }^{116} \mathrm{Sn}$ systems, including dynamics actually increased the fusion barrier as the TDHF fusion threshold is higher than the frozen HF bare potential barrier. Therefore, another dynamical effect aside from vibrations must be considered to help explain this observation.

\subsection{Transfer}

Nucleon transfer in reactions acts to balance out the $N / Z$ ratio between the target and projectile. This effect is accounted for in TDHF calculations [33, 54]. The $N / Z$ ratio of ${ }^{116} \mathrm{Sn}$ is 1.32 and the $N / Z$ ratio for the calcium projectiles range from 1 to 1.70 . For this proceeding, the focus is on proton transfer for these systems. Given these $N / Z$ values, it would be expected from this preliminary argument that proton transfer would happen in the direction from projectile to target for the lighter systems, and from target to projectile in heavier systems.

Nucleon transfer is incorporated into TDHF calculations and is observed during TDHF reactions of the systems ${ }^{40-54} \mathrm{Ca}+{ }^{116} \mathrm{Sn}$ at centre of mass energies $99 \%$ of the fusion threshold for both protons and neutrons. Proton transfer probabilities are calculated using the particle number projection method [31]. The probabilities for the different $Z$ number of the target-like fragment for the systems ${ }^{40,54} \mathrm{Ca}+{ }^{116} \mathrm{Sn}$ can be seen in Fig. 4.

For ${ }^{40} \mathrm{Ca}+{ }^{116} \mathrm{Sn}$, there is a large probability for finding $Z=51$ in the target-like fragment. This corresponds to a likelihood of one proton transfer from projectile to target. By contrast, proton transfer in the opposite direction is likely to occur for ${ }^{54} \mathrm{Ca}+{ }^{116} \mathrm{Sn}$. This is consistent with what is seen in the TDHF fusion barrier thresholds. Proton transfer in either direction will change the Coulomb repulsion. In particular, one proton transfer from ${ }^{54} \mathrm{Ca}$ to ${ }^{116} \mathrm{Sn}$ increases the Coulomb potential, $V_{C}=e^{2} Z_{1} Z_{2} / r$ by $2.7 \%$ which in turn increases the fusion barrier. This is a possible explanation for why the dynamical TDHF fusion threshold is higher than the static frozen HF barrier for this system.

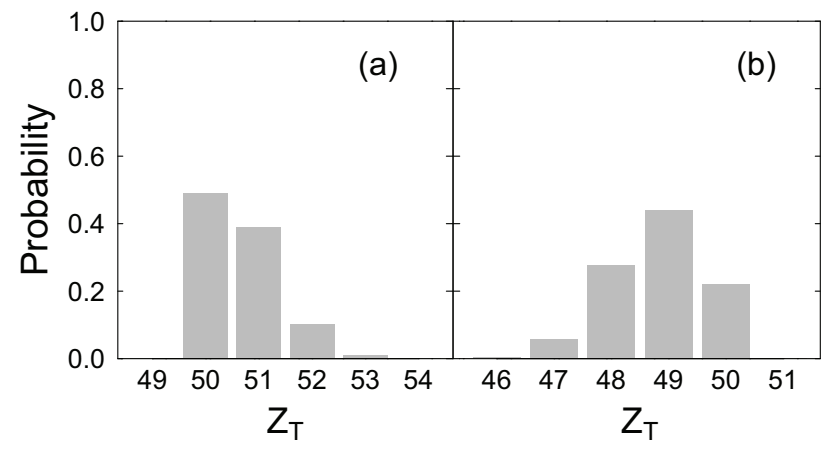

Figure 4. TDHF proton number probabilities for the targetlike fragment in the exit channel of (a) ${ }^{40} \mathrm{Ca}+{ }^{116} \mathrm{Sn}$ and (b) ${ }^{54} \mathrm{Ca}+{ }^{116} \mathrm{Sn}$ reactions at $E_{c m}=0.99 V_{B}$.

\section{Conclusions}

Microscopic theory provides unique insight into nuclear structure and subsequently the effects of structure on reactions. In the static picture of fusion, the HF ground state properties such as rms neutron radius of the calcium isotopes have an effect on the fusion barrier. Nuclei with less bound neutrons and an increasing neutron skin (calcium isotopes heavier than ${ }^{48} \mathrm{Ca}$ ) will lead to a more rapid decrease of the fusion barrier. When dynamics are included into reactions, it was seen that for most of the cases this decreased the fusion barrier. However, it increased the fusion barrier for the two most neutron rich systems. Including dynamics into the calculations overrode the static structure effects completely. Coupled-Channels analysis showed that coupling to the $3_{1}^{-}$state of the calcium projectiles lowered the fusion barrier in all cases. Considering other dynamical effects such as proton transfer is important for the most neutron rich systems. Future works will consider the effect of couplings to low-lying quadrupole vibrations as well as the impact of neutron transfer on fusion.

\section{Acknowledgements}

This work has been supported by the Australian Research Council under Grant No. FT120100760. The calculations in this proceedings were done with the assistance of resources from the National Computational Infrastructure (NCI) which is supported by the Australian Government.

\section{References}

[1] N. Rowley, G. R. Satchler and P. H. Stelson, Phys. Lett. B 254, 25 (1991).

[2] M. Dasgupta, D. J. Hinde, N. Rowley et al., Annu. Rev. Nucl. Part. Sci. 48, 401 (1998).

[3] C. R. Morton, M. Dasgupta, D. J. Hinde et al., Phys. Rev. Lett. 72, 4074 (1994).

[4] J. R. Leigh, M. Dasgupta, D. J. Hinde et al., Phys. Rev. C 52, 3151 (1995).

[5] A. M. Stefanini, D. Ackermann, L. Corradi et al., Phys. Rev. Lett. 74, 864 (1995). 
[6] M. Beckerman, M. Salomaa, A. Sperduto et al., Phys. Rev. Lett. 45, 1472 (1980).

[7] A. Stefanini, G. Fortuna, R. Pengo et al., Nucl. Phys. A 456, 509 (1986).

[8] H. Timmers, L. Corradi, A. Stefanini et al., Phys. Lett. B 399, 35 (1997).

[9] C. L. Jiang, K. E. Rehm, B. B. Back et al., Phys. Rev. C 89, 051603 (2014).

[10] C. L. Jiang, A. M. Stefanini, H. Esbensen et al., Phys. Rev. C 91, 044602 (2015).

[11] M. Dasgupta, D. J. Hinde, R. D. Butt et al., Phys. Rev. Lett. 82, 1395 (1999).

[12] A. Chatterjee, A. Navin, A. Shrivastava et al., Phys. Rev. Lett. 101, 032701 (2008).

[13] A. Lemasson, A. Navin, N. Keeley et al., Phys. Rev. C 82, 044617 (2010).

[14] A. Lemasson, A. Navin, M. Rejmund et al., Phys. Lett. B 697, 454 (2011).

[15] J. W. Negele, Rev. Mod. Phys. 54, 913 (1982).

[16] C. Simenel, Eur. Phys. J. A 48, 152 (2012).

[17] K. H. Kim, T. Otsuka and P. Bonche, J. Phys. G: Nucl. Part. Phys. 23, 1267 (1997).

[18] A. S. Umar and V. E. Oberacker, Phys. Rev. C 71, 034314 (2005).

[19] T. Nakatsukasa and K. Yabana, Phys. Rev. C 71, 024301 (2005).

[20] J. A. Maruhn, P. G. Reinhard, P. D. Stevenson et al., Phys. Rev. C 71, 064328 (2005).

[21] C. Simenel and P. Chomaz, Phys. Rev. C 80, 064309 (2009).

[22] I. Stetcu, A. Bulgac, P. Magierski et al., Phys. Rev. C 84, 051309 (2011).

[23] S. Fracasso, E. B. Suckling and P. D. Stevenson, Phys. Rev. C 86, 044303 (2012).

[24] B. Avez and C. Simenel, Eur. Phys. J. A 49, 76 (2013).

[25] G. Scamps and D. Lacroix, Phys. Rev. C 88, 044310 (2013).

[26] C. Simenel, R. Keser, A. S. Umar et al., Phys. Rev. C 88, 024617 (2013).

[27] C. Simenel, M. Dasgupta, D. J. Hinde et al., Phys. Rev. C 88, 064604 (2013).

[28] A. S. Umar, C. Simenel and V. E. Oberacker, Phys. Rev. C 89, 034611 (2014).

[29] G. F. Dai, L. Guo, E. G. Zhao et al., Phys. Rev. C 90, 044609 (2014).

[30] K. Washiyama, Phys. Rev. C 91, 064607 (2015).
[31] C. Simenel, Phys. Rev. Lett. 105, 192701 (2010).

[32] C. Simenel, Phys. Rev. Lett. 106, 112502 (2011).

[33] K. Sekizawa and K. Yabana, Phys. Rev. C 88, 014614 (2013).

[34] K. Sekizawa and K. Yabana, Phys. Rev. C 90, 064614 (2014).

[35] G. Scamps and D. Lacroix, Phys. Rev. C 87, 014605 (2013).

[36] A. Wakhle, C. Simenel, D. J. Hinde et al., Phys. Rev. Lett. 113, 182502 (2014).

[37] V. E. Oberacker, A. S. Umar and C. Simenel, Phys. Rev. C 90, 054605 (2014).

[38] A. S. Umar, V. E. Oberacker and C. Simenel, Phys. Rev. C 92, 024621 (2015).

[39] C. Simenel and A. S. Umar, Phys. Rev. C 89, 031601 (2014).

[40] G. Scamps, C. Simenel and D. Lacroix, Phys. Rev. C 92, 011602 (2015).

[41] P. Goddard, P. Stevenson and A. Rios, Phys. Rev. C 92, 054610 (2015).

[42] P. Goddard, P. Stevenson and A. Rios, Phys. Rev. C 93, 014620 (2016).

[43] P. Bonche, H. Flocard and P. H. Heenen, Comp. Phys. Com. 171, 49 (2005).

[44] T. Skyrme, Phil. Mag. 1, 1043 (1956).

[45] M. Kortelainen, J. McDonnell, W. Nazarewicz et al., Phys. Rev. C 85, 024304 (2012).

[46] K. Bennaceur, P. Bonche and J. Meyer, C. R. Physique 4, 555 (2003).

[47] R. A. Broglia and A. Winther, Heavy Ion Reaction Lecture Notes, Vol. I: Elastic and Inelastic Reactions (Benjamin/Cummings, Reading, MA, 1981)

[48] C. Simenel and B. Avez, Int. J. Mod. Phys. E 17, 31 (2008).

[49] K. Hagino and N. Takigawa, Phys. Rev. Lett. 79, 2014 (1997).

[50] E. Chabanat, P. Bonche, P. Haensel et al., Nucl. Phys. A 635, 231 (1998).

[51] T. Kibedi and R. H. Spear, Atomic Nuclear and Data Tables 80, 35 (2002).

[52] K. Hagino and N. Takigawa, Prog. Th. Phys. 128, 1061 (2012).

[53] K. Hagino, N. Rowley and A. T. Kruppa, Comp. Phys. Com. 123, 143 (1999).

[54] C. Simenel, D. J. Hinde, R. du Rietz et al., Phys. Lett. B 710, 607 (2012). 